\title{
Análisis de la formación pedagógica del profesorado de los Conservatorios Profesionales de Música y de las Escuelas Municipales de Música
}

\author{
Sara Domínguez-Lloria1, Margarita Pino-Juste²
}

Recibido: 28 de enero de 2020 / Aceptado: 31 de octubre de 2020

Resumen. La formación del profesorado resulta una de las variables más importantes para el éxito educativo. El objetivo de este estudio consiste en describir la formación pedagógica inicial y continua del profesorado en el sistema educativo actual y la formación para el cargo de los directores de los Conservatorios Profesionales de Música y de las Escuelas Municipales de Música en España. Para ello se utilizó un diseño mixto basado en el análisis de contenido de la normativa legal estatal y autonómica vigente, una entrevista a personal cualificado de la Administración y un cuestionario a los directores de estos centros ubicados en la Comunidad Autónoma de Galicia. En esta investigación se concluye que, en la actualidad, existe un vacío muy importante en la formación pedagógica del profesorado de música, siendo la docencia la salida laboral más habitual entre estos titulados superiores.

Palabras clave: Música, Formación del profesorado, Conservatorio, Escuela de Música

\section{Analysis of the pedagogical training of the teachers of the Professional Conservatories of Music and the Municipal Schools of Music}

\begin{abstract}
Teacher training is one of the most important variables for educational success. The objective of this study was to describe the initial and permanent training of the teachers of the Professional Conservatories of Music and of the Municipal Schools of Music in Spain. For this, a mixed design was used through the content analysis of the current state and regional legal regulations, an interview with qualified administration personnel and a questionnaire to the directors of these centers located in the Autonomous Community of Galicia. This research concludes that, at present, there is a very important gap in the pedagogical training of music teachers, with teaching being the most common employment opportunity among these higher graduates.
\end{abstract}

Keywords: Music, Teacher training, Conservatory, Music School

Sumario: 1. Introducción. 2. Marco teórico. 3. Método. 3.1. Participantes. 3.2. Participantes. 3.3. Procedimiento y análisis de datos. 4. Resultados. 4.1. Formación inicial del profesorado. 4.2. Formación permanente del profesorado. 5. Discusión y conclusiones. 5.1. Formación inicial. 5.2. Formación permanente. 6. Referencias bibliográficas.

Cómo citar: Domínguez-Lloria, S.; Pino-Juste, M. (2021) Análisis de la formación pedagógica del profesorado de los Conservatorios Profesionales de Música y de las Escuelas Municipales de Múica. Revista Electrónica Complutense de Investigación en Educación Musical 18, 39-48.

\section{Introducción}

La formación del profesorado y de los equipos directivos de los centros es un asunto de sumo interés, sobre todo si lo relacionamos con procesos de innovación educativa o de mejora de la calidad de la enseñanza.

Universidad de Santiago de Compostela

saradominguez.lloria@usc.es

https://orcid.org/0000-0002-4318-3017

2 Universidade de Vigo

mpino@uvigo.es

https://orcid.org/0000-00022551-5903

Rev. electrón. complut. inves. educ. music. 18, 2021: 39-48 
En el ámbito de la enseñanza de la música, tanto en los Conservatorios Profesionales de Música como en las Escuelas Municipales de Música, la formación del profesorado supone una de las grandes temáticas a abordar, siendo muy problemática la falta de formación docente.

Los planes de estudios de los Conservatorios Superiores siguen valorando más la faceta interpretativa-instrumental ya que la mayoría de las materias se centran en la interpretación, en la teoría de la música y en cuestiones analíticas e históricas.

Sin embargo, la realidad demuestra que prácticamente la totalidad de quienes están en posesión del Título Superior de Música ejercen profesionalmente como docentes.

Por tanto, según Riveiro (2014) existe una necesidad de revisar la formación pedagógica inicial del profesorado de las enseñanzas artísticas de régimen especial ya que hasta el 2002 se carecía de formación en este sentido.

En la actualidad, los profesores de música deben realizar el Máster de Formación del Profesorado de Secundaria, pero en dicho máster no existe una formación específica para los docentes de enseñanzas de régimen especial.

Por consiguiente, resulta importante analizar cuál es la normativa legal actual exigida para la formación del profesorado de Música, así como la formación de los directores para ejercer su cargo tanto en los Conservatorios Profesionales de Música como en las Escuelas Municipales de Música.

Los resultados nos permitirán diseñar estrategias formativas tanto en la formación inicial como permanente de estos profesionales.

\section{Marco teórico}

Establecer un marco teórico sobre formación del profesorado en música resulta una cuestión compleja dada la escasez de estudios sobre este tema. Sin embargo, ya en la década de los noventa, Marchesi y Martín (1998) afirman que en ese momento se produce un cambio muy importante en la formación del profesorado, estableciéndose de forma directa una relación entre la cualificación de los docentes y la calidad de la enseñanza. En ese momento se propone llevar a cabo dos medidas: implicar a los docentes en la puesta en práctica de un enfoque educativo constructivista y focalizar la atención en la formación de los docentes, tanto en el nivel inicial como permanente, a través de distintas asignaturas y cursos de capacitación psicopedagógica, curricular y didáctica (Bautista, Torrado, Pozo, y Pérez-Echeverría, 2006).

La finalidad de estas medidas era que los docentes adquiriesen una visión teórica más compleja acerca del proceso de enseñanza-aprendizaje, que a su vez les facilitase aplicar nuevas metodologías, intentando dotar al profesorado de recursos que promoviesen capacidades como la autonomía y la autorregulación en el alumnado. Todas estas variables incrementarían de forma proporcional la motivación o la creatividad (Torrado, 2003).

Desde este enfoque constructivista, tal como señala López-Bernard (2015), el profesor debe pasar a ser un tutor y un guía que, además de ofrecer soluciones a problemas concretos, da opciones y no impone criterios, orienta, estimula y aumenta la receptividad y la capacidad de respuesta del alumnado ante los hechos artísticos; sin olvidar que el estudiante es un ser único en cuanto al establecimiento de su personalidad artística.

El planteamiento de la enseñanza constructivista en la formación musical del docente fomenta el comportamiento experto en los estudiantes en lo que se refiere al procesamiento artístico (Pozo, 2008).

La teoría constructivista centra la enseñanza en aprender a controlar y dominar los procesos mentales y los procedimientos motores de forma coordinada, siendo el propio estudiante quien debe ejercer el control o regulación de su propia acción.

Refiriéndose a la música, las estrategias didácticas que se emplean en este tipo de enseñanza están basadas en el fomento de la reflexión del alumnado sobre su propia práctica, intentando que se desarrolle un aprendizaje de forma autónoma, lo que implica que aprender precisa del uso de estrategias cognitivas, metacognitivas y conceptuales.

Siguiendo a Vilar i Monmany (2004) señalamos las diferentes perspectivas que hablan del papel que desempeñan los docentes en el desarrollo de las habilidades musicales:

- Estudios sobre la adquisición del sentido rítmico (Imberty, 1995).

- Estudios sobre la adquisición del sentido tonal (Zenatti, 1991).

- Estudios acerca de las formas de comprensión de los objetos sonoros (Sloboda Davidson, Howe y Moore, 1996).

- Estudios sobre el análisis de las respuestas emocionales de los sujetos que interactúan con el fenómeno musical (Sloboda et al.,1996).

Desde esta última perspectiva, Sloboda et al. (1996) revela que los estudiantes ven a sus profesores como los responsables de la adquisición de sus habilidades musicales; sin embargo, quizás es más interesante destacar que el alumnado atribuye su interés en el aprendizaje de un instrumento al hecho de haber establecido una buena relación personal con el profesorado. En el mismo estudio se observó que los estudiantes que obtenían los mejores resultados en la adquisición de destrezas instrumentales tenían una percepción de sus profesores como personas afables, relajadas, amistosas y positivas; sin embargo, la percepción de este alumnado sobre lo que debía ser un buen docente centraba las características en una mayor "calidad profesional". 
Es importante tener en cuenta que la formación instrumental se realiza en ratios 1/1, es decir, se imparte la materia de forma individual donde el docente es la otra pieza clave en la interacción. Por eso, autores como Hallam (2001), McPherson (2001) y Hallam et al. (2012) hacen hincapié en la importancia de que el profesorado tenga claro cuáles son los objetivos docentes para que puedan enseñar al alumnado a pensar de forma estratégica y poder aplicar las enseñanzas al estudio individual. Este hecho hará que las habilidades metacognitivas permitan independizar al alumno y hacer que éste tome las riendas de su propio aprendizaje.

Además, Arévalo (2010) opina que otra de las cuestiones fundamentales que intervienen en la motivación para el aprendizaje instrumental son la determinación y la actuación de los docentes que lo imparten y su capacidad para enfocar los intereses del alumnado.

Hemos de tener en cuenta que el alumnado entiende que un buen docente es aquel que los trata como igual, es próximo y tiene en cuenta la importancia de la actividad en la transmisión cultural del conocimiento (Aróstegui, 2000).

Dada la importancia de estas destrezas docentes para que el proceso de enseñanza-aprendizaje se dé de forma efectiva, es fundamental una formación del profesorado en ámbitos como la psicopedagogía y la didáctica (Rodríguez Quiles, 2003).

Por tanto, el objetivo de este estudio consiste en describir la formación pedagógica inicial y continua del profesorado en el sistema educativo actual y la formación para el cargo de los directores de los Conservatorios Profesionales de Música y de las Escuelas Municipales de Música en la Comunidad Autónoma de Galicia.

\section{Método}

Esta investigación está planteada desde un enfoque descriptivo interpretativo mediante un diseño mixto donde se utilizan instrumentos cuantitativos (cuestionario) y cualitativos (entrevista y análisis de contenido legislativo). En este enfoque convergen dos narrativas solapadas: por un lado, las de los sujetos sociales acerca de sus prácticas y, por otro lado, las narraciones que hacemos los investigadores a partir tanto de lo que vemos como de lo que los sujetos nos cuentan acerca de lo que hacen (Pereira Perez, 2011).

Diversos autores señalan la riqueza de utilizar diseños mixtos, ya que, en estos, al combinarse los enfoques cuantitativo y cualitativo, más allá de que puedan poseer un diseño más complejo, se puede contar con las ventajas de ambos enfoques (Hernández, 2003).

\subsection{Participantes}

Para el estudio cuantitativo llevado a cabo en la Comunidad Autónoma de Galicia han sido recogidos los cuestionarios en las cuatro provincias en todos los centros públicos según figuran en el registro de la Consellería de Educación de la Xunta de Galicia. Participaron un total de 55 directores de los centros de enseñanza reglada y no reglada, tanto de los Conservatorios Profesionales (22 participantes) como de las Escuelas Municipales de Música de la Comunidad Autónoma Gallega (33 participantes), lo que representa más de la mitad de la población ( $\mathrm{N}=106)$ y supone una muestra representativa.

Las entrevistas se realizaron a dos personas: se identificó en primer lugar qué sujetos, por su especialización y cargo, podían ser los más idóneos para completar la información obtenida. Se decidió que uno de los sujetos es un cargo de la Administración de la Consellería de Educación de la Xunta de Galicia (P1), y el otro, un miembro del equipo directivo de un Conservatorio Superior de Música que es también profesor asociado de Universidad del Área de Didáctica de la Música (P2).

\subsection{Instrumentos}

Para analizar los textos legislativos de todas las comunidades autónomas de España con el fin de determinar de forma exhaustiva similitudes y diferencias, se ha empleado un análisis de contenido legislativo. También desde una perspectiva cualitativa, se realizaron entrevistas al personal de la Administración Educativa y de la Universidad, con la finalidad de conocer sus opiniones acerca de la formación del profesorado y de la formación de los equipos directivos en los Conservatorios Profesionales de Música y de las Escuelas Municipales de Música.

Las entrevistas semiestructuradas son fundamentales, debido a que existen una serie de argumentos que justifican el uso de entrevistas cualitativas en investigación. Estos argumentos son fundamentalmente que el conocimiento, las interacciones, y las experiencias de las personas son aspectos significativos de la realidad social que investigamos (Manson, 2002). El cuestionario es una herramienta que permite al investigador plantear una serie de preguntas con el fin de recopilar información de forma estructurada sobre una muestra de personas, utilizando una técnica cuantitativa y agregada de las respuestas que sirven para describir la población a la que pertenecen o contrastar a través de la estadística las relaciones que se pueden establecer entre variables que resulten de interés (Ruiz-Olabuénaga, 2007). En este caso el cuestionario se ha empleado para conocer la opinión de los directores de las Escuelas Municipales de Música y los Conservatorios Profesionales de Música tanto de titularidad autonómica como municipal. 


\subsection{Procedimiento y análisis de datos}

El análisis de contenido se realizó sobre la legislación tanto de las enseñanzas regladas como no regladas extraída de la página web del Ministerio de Educación que proporciona acceso a todas las leyes educativas que rigen tanto a nivel estatal como de la legislación de las diferentes comunidades autónomas. La búsqueda de la legislación se ha ido actualizando de forma periódica realizándose la última el 28 de noviembre de 2018. La búsqueda general dio como resultado un total de 38 documentos legislativos actualizados.

Además, también se ha realizado un análisis de contenido de las entrevistas semiestructuradas. Estas se diseñaron con anterioridad partiendo de un guion basado en las variables del estudio, para permitirnos recoger información y a la vez completar la que ya poseíamos, y favorecer la interacción social entre el investigador y todos los sujetos que participaron.

Durante la entrevista, el entrevistador se ceñía al guion establecido secuenciado y guiado de forma oral y presencial. Se han codificado los textos de ambas entrevistas de tal forma que P1 se refiere al profesional de la administración educativa y P2 al profesor universitario y miembro del equipo directivo de un Conservatorio Superior de Música.

Para la elaboración del cuestionario empleado en este trabajo se han diseñado un total de 13 preguntas cerradas (3 de respuesta corta, 2 de respuesta única y 8 de respuesta múltiple) predominando las respuestas cerradas de respuesta múltiple con respecto a las demás.

Las preguntas de respuesta múltiple del cuestionario se han realizado de acuerdo con una escala Likert-5 que dan la posibilidad al participante de escoger entre las cinco alternativas que se plantean. Una pregunta del cuestionario se ha realizado de acuerdo con una escala Likert y que da la posibilidad al participante de escoger entre las cinco alternativas que se plantean. Posteriormente, el cuestionario fue validado teniendo en cuenta la opinión de 7 jueces expertos tanto en el ámbito de la música (3) como en el ámbito de la metodología cualitativa (4). El cuestionario se administró de forma electrónica a través de una herramienta digital para la elaboración de cuestionarios online cuyos datos fueron recogidos entre septiembre de 2018 y febrero de 2019.

Se llevaron a cabo análisis de frecuencias y porcentajes para describir cuantitativa y cualitativamente las cuestiones referidas a la formación del profesorado y definir la opinión de los directores de los Conservatorios Profesionales de Música y de las Escuelas Municipales de Música.

\section{Resultados}

\subsection{Formación inicial del profesorado}

A través del análisis legislativo comprobamos que las leyes educativas actuales: Ley Orgánica 2/2006 de 3 de mayo, de Educación (LOE) y la Ley Orgánica 8/2013 de 9 de diciembre para la Mejora de la Calidad Educativa (LOMCE), especifican en todos los casos qué titulaciones deben poseer los La LOE 2/2006 de Educación, en sus artículos 94 y siguientes, nos indica que, para impartir docencia en Educación Secundaria Obligatoria, Bachillerato, Formación Profesional y Enseñanzas Artísticas es requisito indispensable poseer la formación pedagógica y didáctica de nivel de postgrado.

Si tenemos en cuenta el contenido del art.100 de la LOE 2/2006 los parámetros fundamentales de la formación inicial de este profesorado se definen del siguiente modo:

a. "la formación inicial del profesorado debe ser suficiente para ajustarse a la necesidad de la titulación y la cualificación requerida por la ordenación general del sistema educativo

b. debe garantizar las capacidades para afrontar el futuro que la educación plantea, así como adaptarse a las nuevas necesidades formativas.

c. estar en posesión de la formación pedagógica y didáctica que el Gobierno establezca para cada tipo de enseñanza, depositando en las administraciones educativas la facultad de establecer los oportunos convenios con las universidades para implantar esta formación.” (p.63).

Por tanto, los titulados en música deben acceder a este máster como profesores de secundaria dado que no existe en ninguna universidad española una oferta específica para las titulaciones de conservatorio y escuelas de música. Así, aunque la ley señala la obligatoriedad de esta formación, en realidad ningún Conservatorio o Escuela de Música en la comunidad autónoma gallega la exige hoy en día a sus profesionales.

Recordemos también que, dentro de las titulaciones que permiten el acceso a la profesión docente, podemos hacer una distinción entre dos grandes grupos: las titulaciones específicamente docentes, como son las distintas modalidades del Grado en Educación Infantil o Primaria con sus respectivas menciones, y las titulaciones que previamente no se asocian a la función docente y cuya obtención permite el ejercicio de la docencia en otras etapas educativas como la Educación Secundaria Obligatoria, Bachillerato, Formación Profesional y las Enseñanzas Artísticas. 
Para el ejercicio de la función docente en las Escuelas Municipales de Música es necesario poseer las titulaciones académicas que se correspondan, tales como: Título Superior de Música establecido en el artículo 54 de la Ley Orgánica del 2/2006 del 3 de mayo de educación, Título Profesional de Música establecido en el artículo 540 de la Ley Orgánica del 2/2006 del 3 de mayo de educación, Título de Profesor Superior o Título de Profesor del plan de estudios que regula el decreto 2618/1966 del 10 de septiembre por el que se aprueba el reglamento general de los conservatorios de música, título de profesor o título profesional del plan de estudios que regula el Decreto del 15 de junio de 1942 . La formación de música y movimiento para niños y niñas entre cuatro y ocho años podrá ser impartida por un maestro especialista en Educación Musical, tal y como se especifica en la circular 12/2006 por la que se establecen las titulaciones que deberá poseer el profesorado que imparta docencia en las escuelas de música y danza de la comunidad autónoma de Galicia.

Para ser director de un centro, en la normativa vigente tampoco se especifica nada sobre la formación inicial ni permanente de esta figura. Aunque existen materias de organización de centros educativos en los diferentes planes de estudios de Magisterio o Diseño y Desarrollo curricular o Organización de centros en el Máster Universitario en Profesor de Educación Secundaria Obligatoria y Bachillerato, Formación Profesional y Enseñanza de Idiomas.

La legislación en la LOE 2/2006 de Educación trata estas cuestiones en su Disposición Adicional Novena donde se especifican los requisitos para el ingreso en los cuerpos de funcionarios docentes. En el punto cuatro se hace una referencia específica a los profesores de Música y Artes Escénicas, siendo necesario estar en posesión del título de Doctor, Licenciado, Ingeniero, Arquitecto o título de Grado correspondiente, u otro título equivalente a efectos de docencia. Además, en el caso del cuerpo de profesores de Música y Artes Escénicas, excepto en las especialidades propias de Arte Dramático, es preciso una formación pedagógica y didáctica a la que se refiere el artículo 100.2 de esta ley, así como superar el correspondiente proceso selectivo.

Se contempla igualmente el acceso a los cuerpos de catedráticos de la misma especialidad, determinando que será necesario estar en posesión del título de doctor, licenciado, arquitecto o título de grado correspondiente o cualquier otro equivalente a efectos docentes y de formación pedagógica y didáctica.

\subsection{Formación permanente del profesorado}

Solamente tres comunidades autónomas españolas (Andalucía, Galicia y Castilla la Mancha) hacen referencia en sus legislaciones a la formación permanente del profesorado de los Conservatorios Profesionales de Música. Se afirma que el profesorado de Música debe contar con la debida actualización y formación de forma sistemática. En el caso de Andalucía esta cuestión es tratada en el artículo 19 del capítulo VI del Decreto 241/2007, de 4 de septiembre, por el que se establece la ordenación y el currículo de las enseñanzas profesionales de Música en Andalucía. En el caso de la comunidad autónoma gallega es reflejada esta cuestión en el artículo 21 del capítulo VI del Decreto 203/2007, de 27 de septiembre, por el que se establece el currículo de las enseñanzas profesionales de régimen especial de música. En la comunidad autónoma de Castilla la Mancha aparece en el artículo 12 del capítulo III del Decreto 76/2007, de 19-06-2007, por el que se regula el currículo de las enseñanzas profesionales de Música en la comunidad autónoma de Castilla-La Mancha.

Si analizamos la opinión de los directores de los Conservatorios Profesionales de Música de titularidad autonómica sobre la formación necesaria para el ejercicio de dicho cargo, opinan en un 17\% no tener ninguna formación; el $17 \%$ afirman tener formación a través de cursos de menos de 50 horas; el 50\% tiene formación a través de cursos de más de 100 horas y el $17 \%$ a través de cursos entre 50 y 100 horas impartidos por las administraciones educativas. Consideran que el conocimiento que poseían cuando comenzaron a ejercer el cargo era bajo $(\square=1.83)$, pero contrasta con lo que consideran que poseen en la actualidad $(\square=3.5)$. Además, piensan que el nivel de conocimiento del equipo directivo con el que trabajan es alto $(\square=3.5)$ y que el mayor desconocimiento recae en el claustro $(\square=2.16)$.

Con respecto a la formación de los directores de los Conservatorios Profesionales de Música de titularidad municipal, el 50\% de ellos afirman que no poseen formación específica para el ejercicio del cargo, el $25 \%$ afirma que dicha formación fue obtenida en cursos de más de 100 horas y el 25\% en cursos de entre 50 y 100 horas. De los directores que afirmaban poseer dicha formación, el 50\% fue a través de los sindicatos y el 50\% como formación universitaria.

Los directores afirmaron que el conocimiento que poseían cuando comenzaron a ejercer el cargo era bajo ( $\square=2.58$ ), pero contrasta con lo que consideran que poseen en la actualidad ( $\square=4.33$ ). También consideran que el nivel de conocimiento del equipo directivo con el que trabajan es alto $(\square=3.91)$ y que el mayor desconocimiento recae en el claustro $(\square=2,83)$.

Acerca de la formación de los equipos directivos en las Escuelas Municipales de Música nos encontramos con que el 55\% de los directores afirman no tener ninguna formación; el 9\% la obtuvo en cursos de más de 100 horas; el $6 \%$ en cursos de entre 50 y 100 horas; el $12 \%$ a través de cursos de menos de 50 horas y el $18 \%$ posee formación de máster o formación universitaria específica.

De los directores que poseen formación, el $40 \%$ afirma haberla recibido a través de los sindicatos, el $47 \%$ por formación universitaria y el 13\% la recibió por parte de la administración educativa.

Sobre el conocimiento que poseían cuando comenzaron a ejercer el cargo de director afirman que era muy bajo $(\square=1.42$ ), pero contrasta con lo que consideran que poseen en la actualidad $(\square=3.42)$. Consideran que el nivel de conocimiento del equipo directivo con el que trabajan es más bajo $(\square=2.93)$ que el del claustro $(\square=3.24)$. 
Tabla 1. Descripción de resultados acerca del grado de conocimiento de la regulación administrativa por parte de los directores y el claustro en las EMM.

\begin{tabular}{|c|c|c|c|c|c|c|c|}
\hline ÍTEM & 0 & 1 & 2 & 3 & 4 & 5 & $\mathrm{M}$ \\
\hline $\begin{array}{c}\text { Nivel de conocimiento que los directores poseían en } \\
\text { su acceso al cargo. }\end{array}$ & 9 & 9 & 9 & 4 & 2 & 0 & 1.42 \\
\hline $\begin{array}{c}\text { Nivel de conocimiento que los directores considera- } \\
\text { ban que poseían en la actualidad. }\end{array}$ & 1 & 2 & 5 & 3 & 18 & 4 & 3.42 \\
\hline $\begin{array}{c}\text { Nivel de conocimiento que poseía el equipo directi- } \\
\text { vo con el que trabajaban. }\end{array}$ & 1 & 5 & 6 & 7 & 11 & 3 & 2.93 \\
\hline $\begin{array}{c}\text { Nivel de conocimiento que poseía el claustro de las } \\
\text { labores de la función directiva. }\end{array}$ & 2 & 7 & 6 & 8 & 5 & 5 & 3.24 \\
\hline
\end{tabular}

En las entrevistas realizadas para profundizar sobre los datos cuantitativos, surgen opiniones divergentes entre las personas entrevistadas acerca de la profesionalización del ejercicio de la dirección.

Mientras P1 comparte la opción de la profesionalización, pero acotando que es necesario tener mucho conocimiento del sector y no solo de la traslación de gestores o del mundo de la empresa a centros educativos, y señala "es un terreno peligroso contar únicamente con gestores que no conozcan nada del mundo de la música"; P2 se muestra más contrario haciendo hincapié en que más que profesionalización es importante la capacitación del profesorado para poder ejercer puestos directivos y señalando "que el profesorado debe estar capacitado en cuestiones de gestión y organización para poder ejercer puestos directivos".

La opinión de los entrevistados acerca de la formación didáctica de los docentes de las enseñanzas regladas y en las no regladas en ambos casos coinciden en que resulta muy escasa afirmando que "el profesorado no cuenta con una buena formación de base a la hora de afrontar la tarea docente" (P1) y que "No existen hoy en día garantías en la formación del profesorado de música ya que ésta no es ofertada por los propios centros superiores" (P2).

Ambos entrevistados coinciden en que la formación docente es todavía una asignatura pendiente de las enseñanzas musicales tanto regladas como no regladas haciendo hincapié en que, teniendo en cuenta que la docencia es una de las salidas laborales más obvias, es muy problemática la falta de formación docente y los planes de estudios siguen valorando mucho la faceta interpretativa-instrumental señalando P1 "que claramente la función docente es la salida profesional mas evidente para los instrumentistas" y complementando P2 "que es necesaria la oferta de esta especialización desde los centros superiores".

\section{Discusión y conclusiones}

\subsection{Formación inicial}

Del análisis legislativo podemos concluir que claramente la administración propone como indispensable para el ejercicio de la docencia la capacitación pedagógica en la Ley Orgánica de Educación; sin embargo, no hay un acceso definido para estos profesionales dentro del máster de Formación del Profesorado.

Por tanto, de nuestros resultados se deduce que en ningún caso los parámetros referidos a la formación docente tienen el nivel de exigencia que requieren las enseñanzas de régimen general, sobre todo tomando en consideración que para la impartición de docencia en los Conservatorios Profesionales de Música y pese a estar legislado, la administración no oferta formación específica para el profesorado de los Conservatorios Profesionales de Música.

Por consiguiente, los entrevistados coinciden en que el profesorado no cuenta con formación ni capacitación pedagógica suficiente para la labor que desempeñan y que es fruto de la ausencia de especializaciones en este ámbito por lo que es una cuestión pendiente que se debe abordar.

En esta línea, Teixidó (2005) afirma que una de las cuestiones de máxima importancia para un docente es la formación pedagógica del profesorado, la cual garantiza una mayor rentabilidad de los recursos disponibles en aras de una mayor efectividad de resultados a todos los niveles. Y Vilar i Monmay (2004) afirma que el desarrollo profesional de los docentes es un proceso de formación continuo que incide en su pensamiento y en las acciones que realiza como persona y como profesional, y que este desarrollo y evolución se realiza como miembro de una comunidad educativa por lo que hablamos indiscutiblemente de desarrollo institucional.

El equipo de docentes proyecta siempre un estilo cultural que le es propio, y es en la utilización de los conocimientos didácticos y los planes de mejora donde mejor reflejado puede quedar; de ahí la importancia de la formación pedagógica del profesorado (Medina, 2011).

Por otra parte, Louro y Aróstegui (2003) defienden que el modelo cultural que se reproduce en los Conservatorios profesionales de Música y el modelo de enseñanza que se inculca en ellos, conlleva unas relaciones de tipo circular entre la enseñanza recibida y la reproducida, de tal modo que dependiendo de la identidad musical del profesor con respecto a su concepción acerca de lo que es la música culta, nos encontramos no solo con el aprendizaje de tal concepto de lo que es susceptible de ser considerado como obra de arte sino que además difiere la enseñanza que imparte. 
Es importante para que la formación del profesorado sea eficaz, permitirle reflexionar y dar a conocer su opinión con respecto a las necesidades formativas de las disciplinas, ya que estas afectarán a las oportunidades de aprendizaje del alumnado y a la calidad que de ellas se derive (Marcelo, 2011).

\subsection{Formación permanente}

En relación con la formación permanente, podemos afirmar que en los textos legislativos de todo el Estado español no se hace referencia a la formación del profesorado siendo únicamente un parámetro presente en los textos legislativos de tres CC. AA: Andalucía, Castilla la Mancha y Galicia. Sin embargo, Jorquera (2004) opina que es fundamental que el profesor de instrumento musical tenga la posibilidad de adquirir herramientas de relación y comunicación que le permitan transmitir sus conocimientos, dando la posibilidad al alumnado de vivir el proceso de enseñanza aprendizaje con interés y entusiasmo. Pero, de acuerdo con nuestras conclusiones, Torrado (2003) afirma que en los centros de formación musical este cambio todavía no se ha dado. Las razones son múltiples.

Por un lado, los docentes de instrumento se muestran muy reticentes al cambio y tienen dificultades para asumir nuevos planteamientos educativos (Torrado, 2006). Uno de los factores que podrían haber contribuido en la adquisición de esta reticencia al cambio y a la repetición de modelos adquiridos es que en la actualidad en nuestros centros de formación musical nos encontramos muy alejados de planteamientos constructivistas (Bautista y Pérez-Echeverría, 2008). A pesar de esta situación, Díaz (2012) afirma que el avance en formación del profesorado y el interés por la formación pedagógica en la formación musical está siendo mucho mayor. Hoy en día existen multitud de publicaciones, tesis doctorales y proyectos de investigación realizadas por profesionales de la música que ponen a la didáctica y la pedagogía musical en un punto de vista no apoyado por la intuición y las reflexiones filosóficas, sino en el lugar en el que se obtengan conclusiones a través de procedimientos científicos como los que aporta la investigación sistemática (Díaz, 2012).

Con respecto al conocimiento percibido que poseen los equipos directivos de las funciones propias de esta labor, es mayor en los Conservatorios Profesionales de Música de titularidad autonómica que en los Conservatorios Profesionales de Música de titularidad municipal. Esta situación puede provocar falta de implicación en las labores directivas de los equipos de los Conservatorios Profesionales de Música de titularidad autonómica. De hecho, Rodríguez, Cárdenas y Campos (2012) opinan que es importante que los líderes de los equipos directivos conozcan a fondo las funciones que se les encomiendan, dado que las funciones que lleven a cabo determinan las innovaciones que se realizan en los centros e influyen en la mejora de las organizaciones educativas.

En las Escuelas Municipales de Música los requisitos de titulación que se exigen al profesorado de estos centros no han sido actualizadas en su mayoría y solo algunas de las comunidades autónomas han tenido en cuenta estas cuestiones reorganizando la legislación a nivel autonómico.

Es reseñable que en la formación de los directores para el acceso al cargo en las Escuelas Municipales de Música es la más alta con respecto a los Conservatorios Profesionales de Música, aunque la mayoría no la tiene alrededor de la mitad de los directores de los Conservatorios Profesionales de Música presentan formación de máster o universitaria específica en cuestiones de gestión. Las medias de edades de estos centros también son más bajas y este tipo de formación específica ha proliferado en los últimos años. Se puede deducir de todo ello que el nivel de exigencia formativa para el acceso al mundo laboral es cada vez mayor.

Los directores de los Conservatorios Profesionales de Música afirman tener la tasa de conocimiento cuando accedieron al cargo más baja y también la más baja respecto de su conocimiento en la actualidad. Esto puede ser consecuencia de que la media de tiempo que llevan como directores es inferior a los de los Conservatorios Profesionales de Música, independientemente de su titularidad. El nivel de conocimiento del equipo directivo es bajo. Hay que tener en cuenta que también estos centros son los que tienen menos cargos directivos complementarios y el claustro parece poseer un mayor conocimiento que en los Conservatorios Profesionales de Música. Es indiscutible la importancia de los equipos directivos en cualquier institución; por ello, es importante tener en cuenta la importancia del liderazgo en estos equipos. En esta línea Bolívar (2010), opina que la mejora de los centros depende directamente de los líderes que dinamicen los centros para que éstos aprendan a desarrollarse a través de lo que se puede denominar liderazgo pedagógico; esto es a su vez coincidente con lo que describen Vargas (2010) y Enrique (2012) añadiendo que para poder gestionar una institución educativa es imprescindible tener en cuenta los diferentes estudios, trabajos, capacitaciones y experiencias de los directores de los centros para incidir en las limitaciones y fomentar una mejora de las instituciones educativas.

De todos modos, sí coinciden en cuanto a la capacitación de los directores y su conocimiento del funcionamiento de la dirección Cantero y Pantoja (2016), quienes afirman que a día de hoy la gran cantidad de tareas a las que se enfrenta un cargo directivo, teniendo en cuenta que no existe profesionalización de los puestos directivos, hace que la exigencia de determinadas funciones lleve a los directores a sentirse en la indefinición de la ambigüedad profesional; en la línea de la importancia del liderazgo en los cargos de dirección, autores como Cantón (2013), opinan que la mejora en el liderazgo de los equipos directivos es clave para procurar una mejor educación. Desde este punto de vista, dicha autora recomienda que las políticas de un país debieran ir encaminadas a la selección y formación de los directores de los centros educativos. En la actualidad nos encontramos con que los directores poseen escasa permanencia en el cargo o directamente existe una falta importante de candidatos, dadas las circunstancias a las que 
los equipos directivos se enfrentan. Sarasúa (2013) coincide en estas opiniones con Cantón (2013) y afirma que los diferentes sistemas educativos europeos consideran que la dirección escolar y el liderazgo educativo son, sin duda, un elemento fundamental en la calidad de la enseñanza y la mejora en el funcionamiento y la organización de los centros y de los resultados que de ella se derivan.

Finalmente, uno de los grandes problemas de las titulaciones en Música es la separación existente en las Enseñanzas Artísticas que se desarrollan en los conservatorios y otras titulaciones de ámbito universitario de perfil docente, para los cuales es obligatorio la realización del Máster de Formación del Profesorado. El profesorado perteneciente a centros de formación musical no cuenta con una oferta especializada desde las administraciones públicas, pese a que la legislación le obliga a realizar un máster. Esta conclusión se deriva de que, pese a la obligatoriedad legislativa de estar en posesión del Máster de Formación del Profesorado para el acceso a la función docente, en la práctica al cuerpo de profesores de Música y Artes Escénicas no se les exige como requisito obligatorio.

La principal propuesta de mejora debería centrarse en solicitar a las administraciones que oferten formación pedagógica especializada a los futuros docentes de los centros de formación musical desde las universidades, en el mismo nivel que se establece para el ejercicio de la docencia de los diferentes niveles y especialidades. Por otro lado, resulta de interés, a su vez, establecer planes de formación continua que mejoren la calidad y la formación de los futuros docentes de Música en cuestiones metodológicas y de innovación, para así garantizar que las prácticas docentes en las enseñanzas de régimen especial posean los mismos estándares de calidad y formación que se exige al resto de titulados superiores.

Otra posible opción, que también se ha analizado para la mejora de la formación del profesorado, es la incorporación de los conservatorios de música al ámbito universitario. Pero la universidad no asume las enseñanzas superiores de música por entender en España que las Enseñanzas Artísticas son la prolongación de una enseñanza extraescolar no siendo consideradas como Grados Universitarios. Esto se contradice con lo que sucede en la mayoría de los países europeos, que otorga la misma categoría a todas las enseñanzas superiores. Si en España se adoptara este modelo, se podría abandonar la distinción entre titulaciones superiores de primera y de segunda categoría, logrando que hubiera una titulación genérica de Graduado en Música, acorde a una oferta de especializaciones internas y diversificaciones curriculares (Sobrino y Mato, 2013).

Algunos autores como Álvaro Marías (1999) plantean tres posibilidades para dar una posible solución a la ubicación de los estudios superiores de música. Esto tiene que ver con la formación del profesorado.

Integración en la universidad.

- Constitución de un nuevo modelo universitario que esté separado del resto de las universidades y que integre las Enseñanzas Artísticas en lo que se ha denominado una Universidad de las Artes.

- Permanencia fuera del ámbito universitario, convirtiendo a los conservatorios superiores en institutos superiores de música.

Más complejo resulta el encuadre del profesorado de las Escuelas Municipales de Música que no se encajan en ningún cuerpo concreto, sino que están al amparo de una categoría profesional dependiente de la adscripción al convenio colectivo laboral que decida el contratante. En el Real Decreto 1834/2008 se definen las condiciones de formación para el ejercicio de la docencia en la Educación Secundaria Obligatoria, el Bachillerato, la Formación Profesional y las Enseñanzas de Régimen Especial y se establecen las especialidades de los cuerpos docentes de enseñanza secundaria. Todas las referencias de este Real Decreto son aplicables tanto a los docentes adscritos al sector privado como público.

\section{Referencias bibliográficas}

Arévalo, A. (2010). La motivación en la realidad actual de la educación. Innovación y experiencias educativas 29, 1-14.

Aróstegui, J. L. (2000). Democracia y currículum: la participación del alumnado en el aula de música. [Tesis doctoral no publicada, Universidad de Granada].

Bautista, A. y Pérez-Echeverría, Ma P. (2008). ¿Qué consideran los profesores de instrumento que deben enseñar en sus clases? Cultura y Educación, 1(20), 17-34.

Bautista, A., Torrado, J.A., Pozo, J.I. y Pérez-Echeverría, Mª.P. (2006). Las concepciones de los profesores de conservatorio sobre el aprendizaje y la enseñanza. En M. O. Ortiz, Cultura, Culturas. Estudios sobre Música y Educación Intercultural, 159-177. Granada: GEU.

Bolívar, A. (2010). Liderazgo pedagógico: una dirección para el aprendizaje. Revista del Fórum Europeo de administradores de la educación, 1, 15-20.

Cantero, N y Pantoja, A. (2016). El papel del director de un centro trasnformado en comunidad de aprendizaje desde la perspectiva del profesorado. Aula de encuentro, 2(18), 76-96.

Cantón, I. (2013). Antecedentes, selección, y formación y calidad de los directores escolares. Participación educativa, Revista del Consejo Escolar del estado, 2(3), 165-173. 
Decreto del 15 de junio de 1942 sobre organización de los Conservatorios de Música y Declamación. Boletín Oficial del Estado. Madrid, 4 de julio de 1942, núm 185.

Decreto 76/2007, de 19-06-2007, por el que se regula el currículo de las enseñanzas profesionales de música en la Comunidad Autónoma de Castilla-La Mancha. Boletín Oficial de la junta de Castilla la Mancha. Toledo, 22 de junio de 2007, núm 131.

Decreto 203/2007, de 27 de septiembre, por el que se establece el currículo de las

enseñanzas profesionales de régimen especial de música. Diario Oficial de Galicia. Santiago de Compostela, 31 de octubre de 2011, núm 211.

Decreto 241/2007, de 4 de septiembre, por el que se establece la ordenación y el currículo de las enseñanzas profesionales de Música en Andalucía. Boletín Oficial de la Junta de Andalucía. Sevilla, 14 de septiembre de 2007, núm 184.

Decreto 2618/1966 del 10 de septiembre sobre reglamentación general de los Conservatorios de Música. Boletín Oficial del Estado. Madrid, 24 de octubre de 1966, núm 254.

Díaz, M. (2012). Los trabajos de investigación en el grado superior de música, búsqueda de un criterio común. Relación con la investigación universitaria. En Álamo, A y Molina, M (coord.), CEIMUS II. Barcelona.

Enrique, R. L. (2012). Gestión directoral y el clima institucional en la I.E. Requena. [Tesis doctoral, Universidad Nacional de Educación Gumán y Valle].

Hallam, S. (2001). The Development of Metacognition in Musicians: Implications for Education. British Journal of Music Education , 1(18), 27-39. https://doi.org/10.1017/S0265051701000122

Hallam, S., Rinta, T., Varvarigou, M., Creech, A., Papageorgi, I., Gomes, T y Lanipekun, J. (2012). The development of practising strategies in young people. Psychology of Music, 5(40), 652-680. https://doi.org/10.1177/0305735612443868

Hernández, R. F. (2003). Metodología de la investigación (3a ed.). México: Mc Graw-Hill.

Imberty, M. (2000). Prospettive di ricerca per la didattica musicale. Quaderni della SIEM, 16, 445-453.

Jorquera, M. C. (2004). Métodos históricos o activos en educación musical. Revista electrónica Europea de Música en la Educación, 14, 1-55.

Ley Orgánica 2/2006, de 3 de mayo, de Educación. Boletín Oficial del Estado.Madrid,4 de mayo de 2006, núm 106.

Ley Orgánica 8/2013, de 9 de diciembre, para la mejora de la calidad educativa. Boletín Oficial de Estado. Madrid, 10 de diciembre de 2013, núm 295.

López-Bernad, L. (2015). Programa para el desarrollo de la inteligencia emocional en los conservatorios de música. [Tesis doctoral, Universidad Nacional de Educación a Distancia].

Louro, A. L y Aróstegui, J.L. (2003). Docentes universitários/professores de instrumento: suas concepçoes sobre educaçao e música. Em Pauta, 14 (22), 35-64.

Manson, J. (2002). Qualitative Researching (2. ${ }^{a}$ ed.). Londres: Sage.

Marcelo, C. (2011). La evaluación del Desarrollo Profesional Docente. En Vélaz de Medrano, C. y Vaillant, D. (Coords.). Aprendizaje y desarrollo profesional docente. 119-127. Madrid: Fundación Santillana/OEI

Marchesi, A y Martín, E. (1998). Calidad de la enseñanza en tiempos de cambio. Madrid: Alianza Editorial.

Marías, A. (1999). ¿La Música en la Universidad?. Revista Cuenta y Razón, 2-3.

McPherson, G. R. (2001). A longitudinal study of self-regulation in children's musical practice. Music Education Research, 2(3), 169-186. https://doi.org/ 10.1080/14613800120089232

Medina, A. (2011). El ambiente simbólico. La cultura del centro educativo. En M. Lorenzo, Organización y gestión de centros y cointextos educativos (pp. 397-422). Madrid: Universitas S.A.

Pereira Perez, Z. (2011). Los diseños de método mixto en la investigación en educación: Una experiencia concreta. Revista Electrónica Educare, 14(1), 15-29. https://bit.ly/3xY0coB

Pozo, J. I. (2008). El aprendizaje y la enseñanza de la interpretación musical: Cambiando las concepciones y las prácticas. Cultura y Educación, 1(20), 5-15. http://dx.doi.org/10.1174/113564008783781495

Riveiro, L.E. (2014). El conocimiento pedagógico y didáctico del profesorado de las Enseñanzas Artísticas Musicales. DEDiCA. Revista de Ediçao e Humanidades, 6, 295-308. https://doi.org/10.30827/dreh.v0i6.6981

Rodríguez Quiles, J. A. (2003). ¿Es necesaria una educación musical para todos?. Revista electrónica de LEEME, 12, 1-5.

Rodríguez, S., Cárdenas, C., Campos, F (2012). El desarrollo del liderazgo educativo. Evidencias de un estudio de historias de vida. REICE. Revista Iberoamericana sobre Calidad, Eficacia y Cambio en Educación, 1(10), 44-57.

Ruiz-Olabuénaga, J. L. (2007). Metodología de investigación cualitativa. Bilbao: Universidad de Deusto.

Sarasúa, A. (2013). La dirección escolar. Luces y sombras. Padres y maestros, 350, 41-44.

Sloboda, J. A., Davidson, J. W., Howe, M. J. A y Moore, D.G. (1996). The role of practice in the development of performing musicians. British Journal of Psychology, 87 (2), 287-309. https://doi.org/10.1111/j.2044-8295.1996.tb02591.x

Sobrino, M., y Mato, P. (2013). Las titulaciones de música en el EEES: Adaptación de los nuevos planes de estudios al nuevo panorama educativo. Musicrearte Digital, 1 (2), 7-11.

Teixidó Saballs, J. (2005). Los centros educativos como organizaciones. Recuperado de: http://www.joanteixido.org/doc/org-educat/centro_como_organizacion.pdf

Torrado, J. A. (2003). Las concepciones de profesores de instrumento sobre el aprendizaje de la música. Un estudio sobre la enseñanza de instrumentos de cuerda en los conservatorios profesionales. [Tesis doctoral no publicada, Universidad Autónoma de Madrid]. 
Torrado, J. A. (2006). Del dicho al hecho: De las concepciones sobre el aprendizaje a la práctica de la enseñanza de la música. En J. I. Pozo, Nuevas formas de pensar la enseñanza y el aprendizaje. Las concepciones de profesores y alumnos (pp. 205-228). Barcelona: Graó.

Vargas, D. (2010). Gestión pedagógica del trabajo docente a través de grupos cooperativos. [Tesis doctoral, Universidad Católica del Perú].

Vilar i Monmany, M. (2004). Acerca de la Educación Musical. Revista LEEME, 13, 1-25. Recuperado de https://ojs.uv.es/index. php/LEEME/article/view/9748/9182

Zenatti, A. (1991). L'enfant et son environnement musical. Issy-les-Moulineaux: E.A.P. 